Luigi Ferrajoli. Catedrático de Filosofía del Derecho y de Teoría General del Derecho en la Universidad de Camerino (19702003) y en la Università degli Studi di Roma Tre; es uno de los principales teóricos del garantismo jurídico. Autor de trescientas publicaciones, entre ellas veinte libros, dentro de los que se destacan: Diritto e ragione. Teoria del garantismo penale, Laterza, Roma-Bari, 1989; Diritti fondamentali. Un dibattito teorico, a cura di Ermanno Vitale Roma-Bari, Laterza, 2001; Principia Iuris. Teoria del diritto e della democrazia, vol. 1 e 2, Laterza, Roma-Bari, 2007; La democrazia attraverso i diritti, Laterza, Roma-Bari, 2013; La logica del diritto. Dieci aporie nell'opera di Hans Kelsen, RomaBari, Laterza, 2016.

Contacto: 1.ferrajoli@uniroma3.it 


\title{
EL PROCESO DE PAZ EN COLOMBIA Y LA JUSTICIA PENAL TRANSICIONAL
}

\author{
Luigi Ferrajoli
}

Università degli Studi Roma Tre

\section{THE PEACE PROCESS IN COLOMBIA AND TRANSITIONAL PENAL JUSTICE}

DOI: $1017450 / 160202$

Fecha de recepción 28 de septiembre de 2016; fecha de aceptación 11 de octubre de 2016. El artículo es fruto de un proyecto de investigación desarrollado en el Dipartimento de Giurisprudenza de la Università degli Studi Roma Tre.

\section{Resumen}

El presente artículo aborda, a partir de un cuestionamiento general al proceso de refrendación vía plebiscito de los acuerdos de paz suscritos entre el Gobierno de Colombia y la guerrilla de las FARC-EP, la naturaleza de la paz como imperativo social y normativo. En primer lugar, se realiza una aproximación a la justicia transicional como herramienta ideal para fomentar los procesos de reconciliación y, en segundo lugar, se 
exponen dos componentes que se proponen como garantías para la construcción de paz: la afirmación del monopolio legítimo de la fuerza por parte del Estado y con ello el favorecimiento al rechazo a las armas, y el desarrollo de la democracia en sus dimensiones política o formal y social o sustancial.

\section{Palabras clave}

Colombia, paz, justicia transicional, reconciliación, democracia.

\section{Abstract}

This article addresses from a general questioning of the plebiscite endorsement process to the peace accords signed between the government of Colombia and the FARC-EP guerrillas, the nature of peace as a social and normative imperative. Firstly, an approach to transitional justice is made as an ideal tool to promote reconciliation processes. Secondly, two components are proposed as guarantees for peacebuilding: the affirmation of the legitimate monopoly of force by part of the State and with it the favoring of the rejection of arms, and the development of democracy in its political or formal and social or substantial dimensions.

\section{Keywords}

Colombia, peace, transitional justice, reconciliation, democracy. 


\section{El valor de la paz}

Lastimosamente, en el referéndum que tuvo lugar en Colombia el pasado 2 de octubre sobre el acuerdo de paz entre el Estado y los guerrilleros de las FARC prevaleció el No, aunque por muy pocos votos: 6.419 .759 (50,21\%) contra 6.359 .643 (49,78\% de los votantes). Además, los votantes solo representaban el 37,43\% de los electores. Así las cosas, al acuerdo de paz se opuso menos del $19 \%$ de todo el electorado, en el que influyó también una inescrupulosa campaña a favor del No, basada en mentiras y dirigida a alimentar rencores y miedos.

Sin embargo, el resultado negativo del referéndum -esta es la tesis que queremos apoyar- no puede comprometer el proceso de paz. El principio de la paz-así como tal vez más de otros principios fundamentales, como la dignidad de la persona, la igualdad, las libertades y los demás derechos humanos- representa una precondición de la convivencia civil. Por ende, el principio de la paz tiene un carácter antimayoritario, en el sentido de que no necesita del consenso de la mayoría ni tampoco puede ser anulado por la voluntad de una mayoría cualquiera. Por eso, tal y como hemos afirmado también en otras ocasiones, el acuerdo de paz no se debía someter a referéndum, porque este no era y no es necesario para legitimar la paz, así como no era suficiente para deslegitimarla.

En efecto, la paz es el valor político supremo, prejudicial a cualquier otro, incluida la misma democracia, las libertades fundamentales y los demás derechos humanos, puesto que ella representa la premisa necesaria de todos los otros valores políticos ${ }^{1}$. Además, en Colombia, el carácter contramayoritario de la paz como fundamento de la convivencia nacional ha sido constitucionalizado. El artículo 22 de la Constitución colombiana establece que "La paz es un derecho y un deber de obligatorio cumplimiento". A diferencia de otras constituciones que también incluyen -como la italiana- el rechazo a la guerra, la Constitución colombiana presenta la paz como un derecho fundamental de la persona y, al mismo tiempo, como un deber de la esfera pública con el que el Estado tiene que cumplir; de ahí el carácter absoluto e incondicional de ese derecho y la inderogabilidad

\footnotetext{
1. En el prólogo de su interesantísimo libro de 1944, La paz por medio del derecho, Kelsen sostiene que hay verdades tan evidentes que hay que repetirlas más veces para evitar que se olviden. La verdad es que la guerra es un homicidio de masas, la peor desgracia de nuestra cultura. Garantizar la paz debe ser el objetivo principal y mucho más importante que la elección entre democracia y dictatura, o entre capitalismo y socialismo. H. Kelsen, La paz por medio del derecho, Editorial Losada, Buenos Aires, 1946. Ya tres siglos antes del cese del bellum omnium contra omnes típico del estado de naturaleza y la transición del estado de guerra al estado civil, la paz había sido identificada por Thomas Hobbes como el fundamento elemental del contrato social y la razón misma del artificio jurídico e institucional, que consistía en garantizar la vida contra la ley salvaje del más fuerte existente en el estado de naturaleza (T. Hobbes, De cive, Clarendon Press, Oxford University, 1984).
} 
de la obligación que el Estado tiene de ponerlo en práctica incluso contra la voluntad de cualquier mayoría, y de ahí también la impropiedad del referéndum sobre la paz. Sobre la paz no se vota ni se decide por mayoría, así como no se vota ni se decide por mayoría sobre la dignidad de la persona, o la igualdad de las personas o sobre otros principios esenciales, como el derecho a la vida y a las libertades fundamentales.

Si esto es verdad, debemos reconocer que el resultado del referéndum no elimina la obligación constitucional del Estado colombiano de realizar la paz. Además, es evidente que -en términos realistas- la puesta en práctica del imperativo de la paz solo puede efectuarse con base en los complicados acuerdos alcanzados durante la larga negociación de La Habana: el desarme de los combatientes, su inserción en la vida civil y política y, sobre todo, las normas sobre la llamada justicia transicional dirigidas a los excombatientes y decididas con base en el "Marco jurídico para la paz" aprobado por el Congreso colombiano el 14 de junio de 2012 y en el Acto Legislativo 01 de 2012 emitido el 31 de julio de ese año².

En efecto, una evidente condición de la paz es la renuncia del Estado a someter a los contendientes a la justicia penal ordinaria, y la estipulación de un modelo de justicia diferente para ellos: el de la "justicia transicional”, llamado así por el carácter transitorio -la transición del estado de guerra al estado de paz- de las normas que la reglamentan. Precisamente, la justiciabilidad de todos los que cometieron crímenes graves -es decir, cómo son tratados en las formas del derecho, aunque de transición, en lugar de aquellas de la guerra- transforma a los combatientes y a los enemigos de ayer en los ciudadanos y conciudadanos de mañana, realizando de tal manera un rasgo distintivo de la paz.

\section{La justicia transicional}

¿En qué debe consistir esta justicia transicional que, en 2012, en el Congreso colombiano, se imaginaba como el resultado de un acuerdo de paz entre los protagonistas del conflicto? Debe consistir en un tipo de justicia que tiene la finalidad de una real reconciliación nacional, que no implique odios, rencores, sentido de la injusticia

\footnotetext{
2. Sobre esta noción, solo nos limitamos a recordar cuatro recopilaciones de ensayos: Justicia transicional. Teoría y praxis, a cargo de Camila de Gamboa Tapias, Editorial Universidad del Rosario, Bogotá, 2006; International Crisis Group, "Justicia transicional y los diálogos de paz en Colombia”, en Informe sobre América Latina, 49, Belgium, 2013; Diálogo constitucional para la paz. Memorias del IX Conversatorio de la jurisdicción constitucional de Colombia, 18-20 de septiembre de 2013, a cargo de J. I. Palacio Palacio, I. H. Escrucería Mayolo y L. A. Ramírez Álvarez, Editorial Universidad del Rosario, Bogotá, 2014; Dinámicas de posconflicto, supuestos para la paz, a cargo de M. A. López Viveros, Universidad Libre, Bogotá, 2016.
} 
sufrida o deseos de venganza que lleven al peligro de otras guerras. De ahí que este tipo de justicia posea algunas características que lo distinguen de las formas de la justicia penal ordinaria.

El primer rasgo característico consiste en un límite de tipo jurídico. Las violencias entre armados llevadas a cabo en el estado de guerra no se pueden evaluar y calificar en el ámbito del derecho penal ordinario. "Silent enim leges inter armas", escribió Cicerón. De hecho, se trata de violencias inter pares e inter armas, a las que los combatientes están obligados por el estado de guerra. La justicia penal solo puede juzgar los crímenes de guerra y los crímenes contra la humanidad que, en cambio, están caracterizados por la asimetría entre criminales y víctimas, puesto que siempre implican violencias ya no inter armas e inter pares, sino más bien contra sujetos más débiles, por lo general inermes, como lo son las poblaciones civiles o los prisioneros de guerra. Luego hay otro elemento fundamental, que también es un límite -aunque más propiamente político- de la justicia transicional, es decir, la exclusión del arresto por esos crímenes, porque el arresto entorpecería de hecho una real pacificación, aunque quede establecida la necesidad del proceso y la comprobación de la verdad, debidas a las víctimas y a la necesidad de evitar represalias.

En este sentido, la justicia transicional se configura como un tipo de justicia de carácter no "retributivo", no "punitivo", sino simplemente "reparador" y "restaurador". De hecho, este tipo de justicia ha sido llamado "justicia restauradora" cimiento de que valores morales y políticos no menores que el de la justicia retributiva y del severo castigo de los culpables representan la reconciliación nacional y la reparación de las injusticias, debido, sobre todo, a la reconstrucción de la verdad histórica por medio de la palabra de las víctimas, del público reconocimiento y de la indemnización de sus sufrimientos, esto es, de la rehabilitación de su dignidad como personas.

Está claro que dicha justicia transicional -dirigida a la verificación de la verdad en orden a la responsabilidad de los que han cometido aquellas atrocidades y a la indemnización de las víctimas- no equivale a la impunidad procedente de una amnistía política generalizada e incondicionada. Se ha notado que similar amnistía contrastaría con los principios del derecho internacional humanitario, en particular con la exclusión de la impunidad de los crímenes de guerra y de los crímenes contra la humanidad y con la afirmación de su imprescriptibilidad, enunciadas respectivamente por el preámbulo y por el artículo 29 del estatuto de la Corte Penal Internacional aprobado en Roma en

3. F. Cortés Rodas, "El derecho internacional penal y el asunto de la amnistía. El caso de las FARC", en Araucaria. Revista ibero-americana de Filosofía, Política y Humanidades, año 15, 30, Editorial Universidad de Sevilla, 2013, pp. $189-216$. 
$1998^{4}$. Sin embargo, no es esta la razón que convierte en inaceptable la impunidad sucesiva a una posible amnistía. En efecto, desde un punto de vista jurídico, es el mismo derecho internacional el que permite una excepción a la prohibición de la impunidad, si esto ocurre para alcanzar la paz; el artículo 6, apartado 5 del segundo protocolo adicional de 1977 al Convenio de Ginebra de 1949 (dedicado a la "protección de las víctimas de los conflictos armados sin carácter internacional”), establece claramente que:

A la cesación de las hostilidades, las autoridades en el poder procurarán conceder la amnistía más amplia posible a las personas que hayan tomado parte en el conflicto armado o que se encuentren privadas de libertad, internadas o detenidas por motivos relacionados con el conflicto armado ${ }^{5}$

Además, el principio de la paz es la norma fundamental y la base del derecho internacional nacido con la institución de la ONU, de ahí que fuera insensato que la paz fuera obstaculizada por normas de derecho internacional.

Así las cosas, no fue por razones jurídicas, sino por razones morales y políticas que la pura impunidad fue considerada inaceptable en la negociación de paz. Precisamente, la consecución de la paz y la garantía del "nunca más" a la guerra excluyen la tabula rasa, la eliminación, el sobreseimiento y el olvido del pasado. De hecho, la impunidad no solo produciría un sentido de injusticia sufrida y no constatada y una violencia más en perjuicio de las víctimas, sino también el no reconocimiento por parte de estas de los autores de los crímenes como conciudadanos y, lo que es peor, el autodesconocimiento de las mismas víctimas como conciudadanos y una ulterior lesión de su dignidad como personas. Esto sería un grave obstáculo para la paz.

Por el contrario, la pacificación exige la tendencial reconciliación, que solo es posible con la indemnización moral de las víctimas, caracterizada antes que todo por el

\footnotetext{
4. Véase a este propósito N. Chaparro Castañeda, Amnistía e indulto en Colombia, Universidad Nacional de Colombia, Bogotá, 2013, que contiene un análisis crítico de las 17 leyes sobre amnistía e indulto, de las que ocho salieron entre 1965 y la Constitución de 1991 (ibid., pp. 72-101), nueve fueron sucesivas a este período (ibid., pp. 101-132), pero ninguna de estas llevó a la pacificación.

5. Es la norma recordada -en apoyo a la legitimidad de los "perdones judiciales" dirigidos, como en Colombia, a poner punto final a una guerra civil- por F. Cortés Rodas, El derecho internacional penal y el asunto de la amnistía, op. cit., que también habla del art. 150, n. ${ }^{\circ} 17$ de la Constitución colombiana, con base en el cual el Congreso puede "conceder, por mayoría de los dos tercios de los votos de los miembros de una y otra Cámara y por graves motivos de conveniencia pública, amnistías o indultos generales por delitos políticos. En caso de que los favorecidos fueran eximidos de la responsabilidad civil respecto de particulares, el Estado quedará obligado a las indemnizaciones a que hubiere lugar”. Cortés Rodas añade justamente que lo que el estatuto de la Corte Penal Internacional ha querido excluir son las amnistías concedidas en los casos de complicidad de los gobiernos con los criminales, es decir, las "leyes de punto final", las "amnistías en blanco" y las "autoamnistías" creadas para favorecer a los mismos gobernantes.
} 
reconocimiento público de todos los crímenes de guerra sufridos por estas durante los conflictos armados. Para que la guerra no se repita, la pacificación exige la construcción de una memoria colectiva y compartida de los horrores del pasado: no exige el perdón -que no se puede pedir a las víctimas ni puede ser concedido por el Estado-, pero sí la estigmatización colectiva y pública de los crímenes cometidos. Para eso sirven los procesos contra quienes hayan cometido crímenes de guerra o contra la humanidad, y hablamos de procesos más que de penas, de la comprobación de la verdad promovida por las víctimas o por sus parientes y basada en su testimonio más que de ejecución penal, de la reconstrucción histórica de las matanzas más graves y de sus responsabilidades más que de sanciones. En efecto, la finalidad y el objetivo de los procesos es poner punto final a la espiral de la venganza, que de otra manera se haría incesante. Recordemos las conclusiones del ciclo de las tragedias de la Orestíada: la infinita espiral de la venganza de la sangre acaba con la institución, en Atenas y por parte de Atenas, del Areópago, es decir, de un juez tercero que debe constatar las responsabilidades y sustituir la lógica de la guerra y de la venganza con la lógica, opuesta y totalmente asimétrica, del derecho y de la pena ${ }^{6}$.

Está claro que es díficil alcanzar el justo equilibrio entre necesidad de justicia $-y$ de verdad-y necesidad de reconciliación nacional -y de pacificación-. Sin embargo, cabe admitir que una gran lección de civilización -desde una doble perspectiva que podría ser seguida y perfeccionada por Colombia- procede de los Tribunales de la Verdad instituidos en Sudáfrica después del apartheid, y luego en Perú y Guatemala tras otros tantos conflictos internos. Justamente, para conciliar paz y justicia, lo importante es la constatación pública a través de los procesos y no tanto de las acciones de guerra, inter arma. Esto se debe a que no pueden ser sometidas al juicio penal sino solo al histórico, que corresponde al de los crímenes de guerra cometidos por todos los protagonistas del conflicto, es decir, no solo por los guerrilleros y los paramilitares, sino también por parte de los exponentes del ejército regular que hayan cometido violencias, matanzas y torturas para dañar las poblaciones civiles. Es más, los crímenes de estos últimos son aún más graves que aquellos cometidos por los guerrilleros, debido a que proceden de miembros de las Fuerzas Armadas que violan con clamor el derecho penal del mismo Estado que representan.

6. Es el momento de la transición de la justicia privada de la venganza a la justicia pública del derecho penal, celebrado en las Euménides de Esquilo. Sobre esta contraposición entre justicia penal y venganza, entre derecho y guerra, véase nuestro Diritto e ragione. Teoria del garantismo penale, Laterza, Roma-Bari, 1989, 10. a ed., 2011, \$25, 1, pp. 332-336. 
En lo que respecta a las penas que pueden ser impuestas por la justicia transicional, estas pueden ser muy blandas, incluso simbólicas. Para que esta justicia alternativa sea aceptada por todos los contendientes y sea un factor de paz, las penas no deben consistir en la reclusión, sino en penas alternativas a la cárcel, como el arresto domiciliario, la prohibición de ausentarse del lugar de residencia o de permanecer en lugares determinados, la obligación de desempeñar trabajos socialmente útiles, la destitución de funcionarios públicos comprometidos en los crímenes demostrados, etc. En todos los casos -incluso en el posible caso de una amnistía llamada “impropia”, o sea, sucesiva al juicio y a la condena, o de un gran indulto de las penas-, si hay comprobación de la verdad e indemnización de las víctimas, no se podrá hablar de “impunidad”. De hecho, los responsables de un crimen siempre se descubren con la comprobación pública de sus culpas y con la condena y la censura moral y política de su actuación. A esta condena pública -si y solo si se comprobara la verdad-, se añadirá aquella inflexible y cierta de la historia.

Solo una justicia transicional dirigida a la reconciliación y a la búsqueda de la verdad puede asegurar una efectiva pacificación nacional. Ya hemos hablado del papel de paz que ha desempeñado el proceso penal como alternativa a la incesante espiral de la venganza, expresado por el mito de la institución en Atenas de un juez tercero capaz de comprobar la verdad y de sustituir a la lógica de la guerra aquella asimetría del derecho. Entonces, precisamente porque está caracterizada por la renuncia a penas privativas de libertad muy duras, la justicia transicional es un poderoso factor de paz, mayor que el de la justicia penal. En efecto, esta representa de forma más explícita y radical la asimetría y la diferencia entre la civilización del derecho y el incivismo de la guerra, entre Estado de derecho y violencia extralegal; se trata de una asimetría y de una diferencia que representan el factor principal de deslegitimación moral y política de la violencia de la guerra y de aquella de la criminalidad. Por lo tanto, más que penas severas y evidentes diferencias entre la justicia del buen proceso y la injusticia de la violencia arbitraria, la comprobación de la verdad con las garantías penales y procesales del Estado de derecho logra aislar socialmente la violencia salvaje de cualquier tipo y poner punto final, tal y como en la Orestíada, a la espiral de venganza y guerra, que de otra manera, sería incesante.

Sin embargo, para lograr la pacificación y para mantener y consolidar la paz, la justicia penal transicional es insuficiente, aunque es absolutamente necesaria. En nuestra opinión, una política de paz que sirva para reconstruir la convivencia civil sobre la seguridad y la solidaridad debe partir de dos objetivos fundamentales que se explicarán en los dos apartes que se presentan a continuación: primero, el radical rechazo de las 
armas y la afirmación del monopolio estatal de la fuerza; segundo, el desarrollo de la democracia, tanto de las formas de la democracia representativa como de los contenidos garantistas impuestos por la democracia constitucional.

\section{Otra garantía esencial de la paz: el radical rechazo de las armas}

El primer objetivo es el efectivo tránsito del estado de naturaleza al estado civil, por medio del desarme completo de los asociados y el monopolio policial de la fuerza, tal y como fueron teorizados por Thomas Hobbes al principio de la modernidad ${ }^{7}$. Está claro que el desarme de todas las formaciones armadas y la entrega de las armas representan una condición esencial impuesta a todos los sujetos del conflicto para beneficiarse de la justicia transicional en lugar de la justicia penal ordinaria. Sin embargo, consideramos que una efectiva garantía del desarme debería consistir en una medida aún más radical que la simple cesión de armas o una restricción de las condiciones del "porte de armas"; debería consistir más bien en la configuración de todas las armas como "bienes ilícitos", de las que deberían ser prohibidos sin ninguna derogación no solo la tenencia, sino también el comercio y la producción.

Esto es algo fundamental sobre lo que se ha insistido muchas veces ${ }^{8}$ y que supera el problema de la pacificación nacional en Colombia. Cada año, en el mundo, hay centenares de millares de homicidios: solo en 2012 hubo 437.000, la mayoría de los cuales fueron perpetrados con armas de fuego; sin contar a los muertos aún más numerosos (casi dos millones) provocados por las muchas guerras civiles o entre Estados. Más de una tercera parte de los homicidios -es decir, 157.000- han sido cometidos en los países de las Américas, en muchos de los cuales existe el libre comercio de las armas, con una media de 16,3 personas asesinadas por cada 100.000 habitantes, casi tres veces más de la media global, que es de 6 personas por cada 100.000 habitantes, y mucho más que en Europa, por ejemplo en Italia donde, a pesar de las mafias, las camorras y los feminicidios, la misma tasa es de 0,9

\footnotetext{
7. T. Hobbes, Leviathan, or the Matter, Forme and Power of Common-Wealth Ecclesiasticall and Civill, trad. it., Leviatano, a cargo de R. Santi, Bompiani, Milano, 2001, cap. XVII, \$13, pp. 281 y 283, donde Hobbes afirma que, si los hombres quieren la paz y la seguridad, solo pueden lograrlo entregando todo su poder y su fuerza a un único hombre o a una asamblea de hombres, el Estado, la latina civitas, a la que los hombres deben su paz y defensa.

8. En L. Ferrajoli, Principia iuris. Teoria del diritto e della democrazia, vol. I, Teoria del diritto, Laterza, Roma-Bari, 2007, $\$ 11.11$, pp. 784-785, hemos definido los “bienes ilícitos" con la acepción D11.33, como todos aquellos bienes de los que están prohibidos la producción y/o la tenencia y/o el comercio, y vol. II, Teoria della democrazia, \$16.9, pp. 521-527, donde hemos identificado en el monopolio jurídico de la fuerza y en la completa eliminación de las armas como bienes ilícitos la principal garantía de la paz. Hemos corroborado esta propuesta en La democrazia attraverso i diritti. Il costituzionalismo garantista come modello teorico e come progetto politico, Laterza, Roma-Bari 2013, \$ 5.5, pp. 211-213.
} 
por cada 100.000 habitantes $^{9}$. Esto ocurre porque en Italia o en Europa -por costumbre y por el control a las armas- nadie anda armado y casi nadie posee armas de fuego (a pesar de que los países europeos están entre los mayores responsables de la producción y de la venta de armas en todo el mundo). Por el contrario, la difusión de armas y la gran facilidad de disponer de ellas implican consecuencias contagiosas: las personas se arman por miedo. Cuanto más aumenta la difusión de las armas, más aumenta el deseo de los individuos de armarse para defenderse. Así las cosas, del miedo y de la inseguridad generalizadas derivan tanto la ruptura de los vínculos sociales como la confianza recíproca, que representan las premisas elementales de la convivencia pacífica y de la misma democracia.

Lo anterior explica el que no se haya realizado - no solo en Colombia, sino también en ningún otro país, tampoco en la comunidad internacional- el desarme de los asociados y el monopolio público de la fuerza, que son las premisas de la convivencia civil. Producción, comercio, tenencia y difusión de armas -mucho más peligrosas y destructivas que aquellas de la época de Hobbes- son el símbolo de una civilización no alcanzada en nuestras sociedades y el principal factor del desarrollo de la criminalidad, de los terrorismos y de las guerras. De ahí que entendamos los pesados condicionamientos ejercidos sobre la política de nuestros gobiernos por parte de los aparatos militares y de los lobbies de las armas, que no quieren que las armas estén prohibidas como bienes ilícitos, ne cives ad arma veniant. De la misma manera, nos explicamos -gracias a la ilusoria e insensata voluntad de potencia de las naciones, vinculada con los intereses de las industrias de armas que son las únicas que se benefician de los gastos militares ${ }^{10}$ - por qué no se ha realizado aquel progresivo pasaje de la comunidad internacional del estado de naturaleza al estado civil, que solo es posible con la afirmación del monopolio jurídico de la fuerza de la ONU, representado por el apartado VII de la Carta de las Naciones Unidas, y con la consiguiente y progresiva superación de los ejércitos nacionales, ya deseada hace dos siglos por Immanuel Kant ${ }^{11}$.

\footnotetext{
9. El país más violento del mundo es Honduras, con una media en 2012 de 90,4 homicidios por cada 100.000 habitantes. Colombia ocupa la décima posición, con 14.670 homicidios y una media de 30,8 matanzas por cada 100.000 habitantes. El país con un número mayor de homicidios, 50.108, es Brasil, que ocupa la posición 15. Con base en los datos ONU, el $95 \%$ de los homicidios lo cometen los hombres, que también representan el $80 \%$ de las víctimas (A. Mencaraglia, "Vuoi davvero rischiare la tua vita? Le nazioni da evitare", en Italiansinfuga.com, 16 de abril de 2014, disponible en www.italiansinfuga. com/2014/04/16/classifica-delle-nazioni-in-base-al-tasso-di-omicidi; "Il posto più violento del mondo", en Il Post, 14 de abril de 2014, disponible en www.ilpost.it/2014/04/14/rapporto-onu-omicidi-mondo).

10. En 2011, estos gastos alcanzaron 1.740 millardos de dólares, es decir, el 2,6\% del PIB mundial (S. Andreis, Le spese militari nel mondo, en AA.VV., Economia a mano armata. Libro bianco sulle spese militari, Sbilanciamoci, Roma, 2012, p. 81). El $43 \%$ de este gasto, es decir, 698 millardos de dólares, es pagado por Estados Unidos. Les siguen China (119 millardos de dólares), Reino Unido y Francia (59,3 millardos), Rusia (58,7 millardos), Japón (54,5 millardos), Arabia Saudita y Alemania (45,2 millardos), India (41,3 millardos) e Italia (37 millardos) (ibid., p. 83).

11. "Los ejércitos permanentes - miles perpetuus- deben desaparecer por completo con el tiempo. Los ejércitos permanentes son una incesante amenaza de guerra para los demás Estados, puesto que están siempre dispuestos y preparados para
} 
Sobre el tema de la seguridad, una política racional y no demagógica debería prohibir radicalmente la producción, el comercio y la tenencia de armas, en lugar de fomentar inútiles agravaciones de pena y reducciones de las garantías penales, como en cambio ocurre en todos nuestros países gracias al imperante populismo penal. Además, se trataría de una prohibición mucho más eficaz que aquella de las drogas, porque la producción y la venta clandestina de armas es mucho más difícil y complicada. Las armas no se cultivan como las drogas. No existen fábricas de armas en las zonas del mundo más plagadas por guerras, terrorismos y crimen organizado. Prohibir seriamente el comercio y la tenencia de armas sería la primera garantía de la paz, de la seguridad y de la vida, incluso en países como los europeos, donde el número de los homicidios es muy bajo, aunque en su mayoría se cometen con armas de fuego.

Insistimos en esta esencial garantía de la paz y del derecho a la vida de masas inmensas de seres humanos, derivados hoy día de una radical prohibición de todas las armas. Está claro que se trata de un problema global, no solo colombiano. Sin embargo, consideramos que, precisamente, el actual proceso de paz podría ser para Colombia la ocasión para convertirse en el primer país del mundo que prohíba radicalmente las armas: su producción, su comercio y su tenencia, sin ninguna excepción, es decir, sin que haya ninguna posibilidad de "porte de armas". Tendría un extraordinario mérito frente a la historia y sería una gran lección de civilización jurídica y política por parte de Colombia a la comunidad internacional, tras su terrible experiencia de 50 años de guerra civil.

A lo anterior añadimos que estas medidas, a causa del papel performativo que siempre ha tenido el derecho en la formación del sentido común, servirían para eliminar la subcultura de la violencia alimentada por la libre y a veces complaciente y fetichista posesión de las armas. Esta subcultura se manifestó en Colombia como concepción militarista de la lucha política: primero, en el militarismo revolucionario y guerrillero; segundo, en el militarismo estatalista y patriótico. La radical prohibición de las armas serviría para promover, en el sentido común, la relación biunívoca entre democracia y

\footnotetext{
combatir. Los diferentes Estados se empeñan en superarse unos a otros en armamentos, que aumentan sin cesar. Y como, finalmente, los gastos ocasionados por el ejército permanente llegan a hacer la paz aún más intolerable que una guerra corta, acaban por ser ellos mismos la causa de agresiones, cuyo fin no es otro que librar al país de la pesadumbre de los gastos militares. Añádase a esto que tener gente a sueldo para que muera o mate parece que implica un uso del hombre como mera máquina en manos de otro -el Estado-; lo que no se compadece bien con los derechos de la Humanidad en nuestra propia persona" (I. Kant, Hacia la paz perpetua [1795], sez. I, \$3). Kant se pregunta qué derecho tiene el Estado de servirse de sus propios súbditos para declarar la guerra a otros Estados, y parece que esto deriva del derecho de poder hacer lo que quiera con lo suyo, o sea, con lo que le pertenece. Sin embargo, este principio de derecho no se puede aplicar al hombre, sobre todo al ciudadano, que siempre se debe considerar como un miembro del poder legislativo. (I. Kant, Principios Metafisicos del Derecho. Librería de Victoriano Suárez, Madrid, 1873, pp. 215- 218).
} 
paz, respecto de la cual las fuerzas de la democracia garantizan el desarrollo pacífico de los conflictos sociales, impidiendo que acaben en conflictos armados.

\section{Otra garantía de la paz: el desarrollo de la democracia política o formal y de aquella constitucional o sustancial}

Así las cosas, reforzar las formas y la sustancia de la democracia, transformar el conflicto armado en conflicto político equivale a instaurar el segundo tipo de garantías de la paz del que hemos hablado antes: la construcción y el desarrollo de la democracia. Para Hans Kelsen:

La democracia es la forma política de la paz social, de la composición de los contrastes, de la recíproca comprensión sobre una línea mediana... que no es aceptada precisamente por todos los que no quieren la paz y lo que es el precio de la paz: el compromiso ${ }^{12}$.

Se trata de un doble compromiso de paz: por un lado está el compromiso político del que habla Kelsen, realizado por el método de gobierno, es decir, por la dimensión formal o política de la democracia tal y como se deriva de la representación de todos los ciudadanos en las sedes decisionales; y por otro lado está el compromiso social, efectuado por los vínculos constitucionales impuestos a la acción de gobierno, es decir, por la dimensión sustancial de la democracia, tal y como se destaca de los principios y derechos fundamentales constitucionalmente establecidos.

El primer papel de mediación pacífica de los conflictos lo asegura la democracia política como forma y método de gobierno basado en la primacía del Parlamento. De hecho, para Kelsen, el Parlamento es la institución que, por medio de la confrontación entre mayoría y minorías, proporciona la posibilidad de un compromiso, es decir, de una superposición de lo que divide a los asociados en provecho de lo que los une, en

12. H. Kelsen, Defensa de la democracia (1932) trad. it. en Sociología de la democracia, a cargo de A. Carrino, Esi, Napoli, 1991, p. 46. También N. Bobbio -en Il problema della guerra e le vie della pace, Il Mulino, Bologna, 1979, pp. 14-15- ha puesto de manifiesto que no tenemos que ir demasiado lejos para encontrar el ejemplo más convincente del método no violento para la resolución de los conflictos sociales, es decir, la democracia que, ya desde su primera aparición, reemplazó la lucha con la discusión, el golpe de gracia del vencedor sobre el vencido con el voto y la voluntad de la mayoría que le permiten al vencido de ayer convertirse en el vencedor de mañana sine effusione sanguinis. 
una palabra, se trata de tolerancia recíproca ${ }^{13}$. Por eso, para Kelsen la mejor forma de democracia es la parlamentaria, la que procede de la adopción del sistema electoral proporcional, porque él considera que todo el procedimiento parlamentario está dirigido a alcanzar una vía intermedia, que exige que todos los grupos políticos queden representados en el Parlamento, dependiendo de su fuerza, si se quiere que el Parlamento represente la situación de hecho de los intereses en conflicto. Por eso se llega al compromiso ${ }^{14}$.

También es verdad que ni en Colombia, ni en los demás Estados de las Américas, la democracia posee la forma parlamentaria deseada por Kelsen, sino aquella presidencial. Sin embargo, el papel de pacificación de la democracia representativa puede ser alcanzado, igualmente, reforzando los poderes del Parlamento respecto de aquellos del poder ejecutivo y previendo lo que Kelsen ha llamado las garantías necesarias para que los distintos intereses de los grupos representados en el Parlamento se tengan en consideración en el debate público ${ }^{15}$. De hecho, lo que importa es la recíproca legitimación de las fuerzas políticas en conflicto por medio de su representación y de una confrontación pública, antes y durante las competiciones electorales y luego en el debate parlamentario. De esta manera, se garantiza la posibilidad de desarrollo de movimientos de oposición y de manifestaciones de protesta social, además de su legitimación como efectivas alternativas de gobierno; se promueve la transformación en partidos políticos de los antiguos grupos de guerrilla, se refuerzan las garantías de todas las libertades fundamentales, de la libertad de imprenta y de manifestación del pensamiento a las libertades de asociación y de reunión; en una palabra, se toma en serio el importante proyecto normativo establecido por la Constitución colombiana de 1991 y se adquieren los derechos fundamentales establecidos en esta -no solo los derechos políticos y los derechos de libertad, sino también los derechos sociales y los de los trabajadores- como normas jurídicamente vinculantes y lleva, por consiguiente, a la introducción de sus garantías con la producción de sus normas de actuación.

Ahora llegamos a la segunda dimensión de la democracia, la sustancial y constitucional, igualmente necesaria, que se añade a la formal y política recomendada por Kelsen para construir una paz duradera. Esta dimensión sustancial de la democracia, que se ha insertado en la formal y política por medio del constitucionalismo rígido de

13. H. Kelsen, El problema del parlamentarismo, en Escritos sobre la democracia y el socialismo, Debate, Madrid, 1988, pp. 85-105.

14. Ibid. y N. Bobbio, “La non violenza è un'alternativa?”, en Id., Il problema della guerra e le vie della pace, p. 203. Bobbio ha destacado que la función de las llamadas reglas del juego es permitir a las diferentes partes políticas rivalizar pacíficamente para alcanzar objetivos que, de otra manera, se alcanzarían de forma violenta. Para eso sirve el método democrático, es decir, para sustituir los medios violentos por aquellos no violentos.

15. H. Kelsen, El problema del parlamentarismo, p. 105. 
la segunda parte del siglo XX y bien determinada por la Constitución colombiana de 1991, con seguridad representa el factor principal y la garantía más eficaz de la convivencia pacífica y de una recobrada unidad y solidaridad nacional. De hecho, sirve para integrar el papel de mediación de los conflictos exclusivamente confiado, en el antiguo Estado de derecho, al compromiso político adquirido cada vez en las formas de la representación, añadiéndole el más relevante compromiso social y constitucional finalmente estipulado con la constitucionalización rígida de los derechos fundamentales y la imposición a las funciones legislativas y de gobierno de las prohibiciones y obligaciones que las garantizan.

En efecto, solo la garantía de la igualdad y de los derechos fundamentales, primeros entre todos los derechos a la subsistencia -la par condicio civium y sus iura paria, tal y como escribió Cicerón hace más de dos mil años- genera la unidad de un pueblo, es decir, el sentido de pertenencia de todos a la misma comunidad política ${ }^{16}$. Y esto es aún más verdadero en países como Colombia, donde las fuertes desigualdades económicas y los elevados niveles de pobreza siempre amenazan con debilitar el sentido de pertenencia de todos los colombianos a la comunidad nacional. En efecto, los excluidos de la sociedad civil y legal siempre se exponen y están dispuestos a ser incluidos en las comunidades inciviles de las asociaciones criminales que, a su vez, están dispuestas a reclutarlos e incluirlos en sus organizaciones ilegales, como mano de obra subalterna. Por eso, la garantía principal de la paz es la igualdad de todos en lo que concierne a los derechos -los iura paria, nadie excluido- realizable con la construcción de un eficiente Estado social fundamentado en una política fiscal de redistribución de la riqueza. Por lo demás, la imposición de esta construcción se deduce de la misma Constitución colombiana que, en el artículo 1, establece que "Colombia es un Estado social de derecho". La construcción de la paz depende de la construcción de la democracia y de las relativas garantías en todas sus dimensiones: política, constitucional, liberal y social.

Traducción de M. Colucciello

\footnotetext{
16. "Quare cum lex sit civilis societatis vinculum, ius autem legis aequale, quo iure societas civium teneri potest, cum par non sit condicio civium? Si enim pecunias aequari non placet, si ingenia omnium paria esse non possunt, iura certe paria debent esse eorum inter se qui sunt cives in eadem re publica. Quid est enim civitas nisi iuris socie tas ci vium?" (Cicerón, De re publica, en "Collezione romana" dirigida por Ettore Romagnoli, Istituto Editoriale italiano, Roma, 1928, lib. I, XXXII, p. 80).
} 